\title{
Synthesis and characterisation of silica aerogel/carbon microfibers nanocomposites dried in supercritical and ambient pressure conditions
}

\author{
Agnieszka Ślosarczyk ${ }^{1} \cdot$ Mikołaj Barełkowski $^{1} \cdot$ Szymon Niemier $^{1}$ • \\ Paulina Jakubowska ${ }^{2}$
}

Received: 7 June 2015/Accepted: 2 July 2015/Published online: 14 July 2015

(C) The Author(s) 2015. This article is published with open access at Springerlink.com

\begin{abstract}
Experimental results on the physical and chemical properties of silica aerogel and its composites with carbon microfibers are reported. The aerogels and silica aerogel/carbon microfibers nanocomposites were prepared via the sol-gel process of organosilicon compound followed by successive washing and drying in different conditions. Part of nanomaterials was surface modified in TMCS/n-hexane mixture in $50{ }^{\circ} \mathrm{C}$ and dried in ambient pressure. Simultaneously, the supercritical drying of aerogels and its nanocomposites in $\mathrm{CO}_{2}$ atmosphere was carried out. The carbon microfibers before usage in silica aerogel composites were chemically treated in nitric acid. The physical properties of silica aerogels were studied by
\end{abstract}

measuring the bulk density, volume shrinkage, porosity, pore volume and thermal conductivity. In the case of pure aerogels dried via supercritical conditions, the structure presented much less contraction during drying and was more resistant to higher temperature. However, the properties of silica aerogels composites with carbon microfibers drying in ambient pressure were better than corresponding composites subjected to supercritical drying. It was shown that simultaneous oxidation of carbon fibres and chemical modification of silica aerogel in TMCS/n-hexane mixture contributed to the stable mesoporous structure of composite with very good physical properties, especially lower shrinkage.
Agnieszka Ślosarczyk

agnieszka.slosarczyk@put.poznan.pl

1 Institute of Structural Engineering, Poznan University of Technology, Piotrowo 5 Str., 60-965 Poznan, Poland

2 Institute of Technology and Chemical Engineering, Poznan University of Technology, Piotrowo 3 Str., 60-965 Poznan, Poland 


\section{Graphical Abstract}

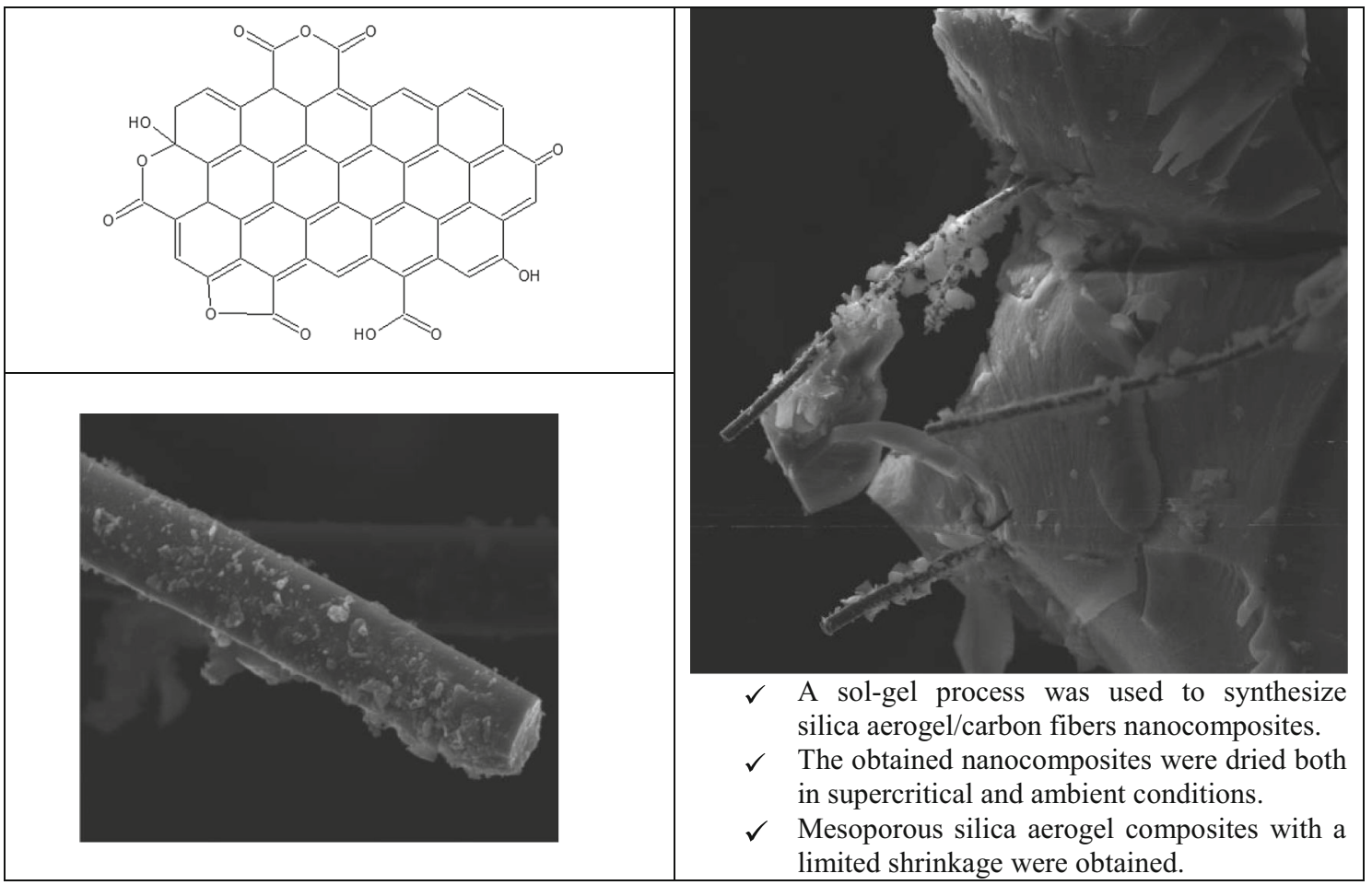

Keywords Silica aerogel - Carbon microfibers - Sol-gel synthesis - Chemical modification - Supercritical and ambient pressure drying $\cdot$ Nanocomposites

\section{Introduction}

Aerogels are characterised by very good compressive strength, but poor fracture toughness, which results from poor flexural and tensile strength. One of the ways for improving this disadvantage is an application of dispersed reinforcement, such as short fibres or directional reinforcement in the form of the mats $[1,2]$. In the literature, little attention is paid to the application of short carbon fibres as silica aerogel reinforcement. Carbon fibres, in comparison with the other fibres, such as polypropylene or glass, are characterised by very good mechanical parameters, especially with regard to their density, very high chemical strength, stable structure up to $750{ }^{\circ} \mathrm{C}$ and very good electrical conductivity [3, 4]. In the following article, the silica aerogels and their nanocomposites with carbon microfibers were synthesised in the double-step sol-gel process, followed by ambient pressure drying as well as drying in supercritical conditions. In order to improve the adhesion between carbon fibre and silica aerogel, the fibres underwent chemical modification in the nitric acid. The structure of the received nanomaterials and the temperature resistance were tested with the following instrumental analysis methods: BET, TG FTIR and SEM.

\section{Experimental}

Precursor for the aerogel synthesis was alkoxide compound, tetraethylorthosilicate (TEOS), in water solution of ethanol. $0.5 \%$ water solution of hydrochloric acid was used as the catalyst of the sol-gel reaction. Chemical

Table 1 Physical and mechanical parameters of carbon microfibers

\begin{tabular}{ll}
\hline Properties & Pitch-based carbon fibres \\
\hline Diameter $(\mu \mathrm{m})$ & 13 \\
Length $(\mu \mathrm{m})$ & 700 \\
Carbon content $(\mathrm{wt} \%)$ & Min. 99 \\
Density $\left(\mathrm{g} / \mathrm{cm}^{3}\right)$ & 1.64 \\
Bulk density $\left(\mathrm{g} / \mathrm{dm}^{3}\right)$ & 290 \\
Resistivity $(\Omega \mathrm{cm})$ & $0.3 \times 10^{-2}$ \\
Moisture content $(\mathrm{wt} \%)$ & 0 \\
Tensile strength $(\mathrm{MPa})$ & 750 \\
Modulus of elasticity $(\mathrm{GPa})$ & 40 \\
Elongation $(\mathrm{mm})$ & 1.9 \\
pH & 7 \\
Temperature resistance $\left({ }^{\circ} \mathrm{C}\right)$ & 750
\end{tabular}


modification of the surface of aerogels and their composites with carbon microfibers was conducted in the solution of TMCS (chlorotrimethylsilane)/n-hexane with the volumetric ratio of $1: 5$ in the temperature of $50{ }^{\circ} \mathrm{C}$ for $48 \mathrm{~h}$. The supercritical drying in $\mathrm{CO}_{2}$ was conducted in the research stand built in Institute of Structural Engineering at Poznan University of Technology, according to the procedure described in the article [5].

Carbon microfibers from carbon pitch of $700 \mu \mathrm{m}$ length and $13 \mu \mathrm{m}$ diameter in the amount of $15 \mathrm{wt} \%$ of silica aerogel mass were used as reinforcement. Parameters of the carbon microfibers are presented in Table 1 . The carbon fibres applied are characterised by very small specific surface area and low degree of surface oxidation; they are practically hydrophobic. Oxidation of the carbon fibre surface was conducted in the liquid phase in nitric acid. Initial fibre sample was placed in the round-bottom flask and poured with concentrated nitric acid. The oxidation process was conducted in the boiling temperature of nitric acid under the reflux condenser for $5 \mathrm{~h}$. Next, the fibres were washed with water to $\mathrm{pH} 7$ and dried to constant weight in the temperature of $105^{\circ} \mathrm{C}$ [6].

For the synthesis of silica gel, two solutions were prepared: solution A consisting of TEOS compound, alcohol and water, and solution $\mathrm{B}$ consisting of reaction catalyst (acidic), alcohol and water. Mixing of the two solutions initiated a hydrolysis reaction, as a result of which sol was created, which was further formed into gel in the process of condensation. Before initiating the gelation, the fibres were mixed in solution $A$ in the magnetic stirrer, then the solution B was added, and within a few minutes, the gelation occurred. After the gelation, the samples were taken for $24 \mathrm{~h}$ to the water solution of alcohol and then for 7 days to pure ethanol. After this time, the samples of the gels and composites gel/modified fibres were put into a mixture of TMCS/n-hexane in the volumetric ratio of $1: 5$ for $48 \mathrm{~h}$ in the temperature of $50{ }^{\circ} \mathrm{C}$, in order to modify their surface. After the modification, the gels were put for $24 \mathrm{~h}$ to a solution of n-hexane and then air-dried until the structure of aerogel was achieved. The silica aerogel was marked as A, and the nanocomposites silica aerogel/modified carbon microfibers relatively as $\mathrm{A}+\mathrm{CFmod}$. In the case of supercritical drying, the samples of aerogel and nanocomposite with modified carbon fibres were dried in
$\mathrm{CO}_{2}$, in the temperature and pressure ensuring the supercritical fluid conditions. The silica aerogel received as the result of the supercritical drying was marked as $\mathrm{B}$, and the nanocomposites silica aerogel/modified carbon microfibers, respectively, as B + CFmod.

For the determination of ATR-FTIR spectrums of the tested samples (silica aerogels and its composites with carbon microfibres), the FTIR NICOLET 5700 machine from Thermo Electron Corporation with ATR attachment (crystal: ZnSe) was used. ATR-FTIR measurement was taken in the wave number range from 600 to $4000 \mathrm{~cm}^{-1}$ (scan number 64), and the spectrums received equalled: $\%$ reflection $=\mathrm{f}$ (wave number).

The structural properties of the received silica aerogels and nanocomposites silica aerogel/carbon microfibers were tested with the use of BET method, on the basis of nitrogen adsorption isotherm $(77.35 \mathrm{~K})$. The ASAP2010 porosimeter from Micrometrix was applied. On the basis of the received adsorption isotherms, the following parameters were defined: the specific surface area and the volume of the micropores of the unmodified and modified carbon fibres, specific surface area and average diameter of the pores for pure aerogels and silica aerogel/carbon microfiber composites. The average pore diameter was calculated on the basis of the $4 \mathrm{~V} / \AA$ equation, where $\mathrm{V}$ stands for total volume of the defined pores in a single point of adsorption isotherm with $p / p_{0}=0.99$.

TGA of the tested materials was conducted in nitrogen, with the use of NETSCH machine, model TG 209 F3. The following measurement parameters were applied: strength of inert gas flow $30 \mathrm{ml} / \mathrm{min}$, rate of sample heating $10^{\circ} \mathrm{C} /$ min, analysis temperature range from 30 to $1000{ }^{\circ} \mathrm{C}$.

\section{Results and discussion}

Table 2 presents the basic physical and structural parameters of the received aerogels and their composites with carbon fibres. Research proved that as a result of drying under supercritical conditions, the same initial formula gives aerogels and their composites with carbon microfiber characterised by lower density and much lower contraction during drying, in comparison with aerogels dried in atmosphere. For pure aerogels dried in supercritical
Table 2 Structural parameters of silica aerogel and its composites depending on drying method

\begin{tabular}{lllll}
\hline Parameter & \multicolumn{2}{l}{ Drying in ambient pressure } & \multicolumn{2}{l}{ Drying in supercritical conditions } \\
\hline Marked in text & A & A + CFmod & B & B + CFmod \\
Density $\left(\mathrm{g} / \mathrm{cm}^{3}\right)$ & 0.20 & 0.13 & 0.125 & 0.10 \\
Surface area by BET $\left(\mathrm{m}^{2} / \mathrm{g}\right)$ & 863.9 & 775.1 & 801.5 & 820.9 \\
Average pore diameter $(\mathrm{nm})$ & 12.2 & 15.8 & 10.7 & 10.9 \\
Volume shrinkage $(\%)$ & 27.7 & 8.8 & 9.1 & 5.9 \\
\hline
\end{tabular}




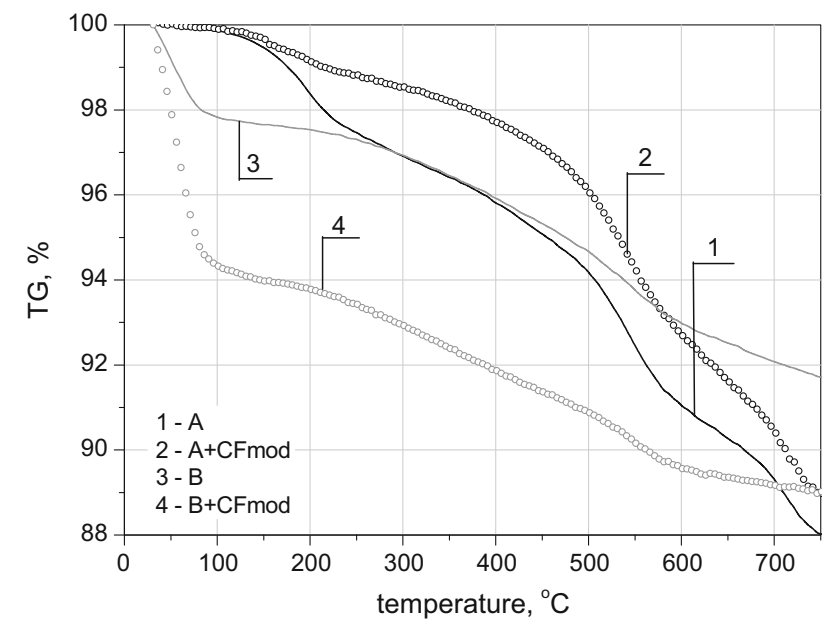

Fig. 1 TGA for pure silica aerogels and its composites with carbon fibres in relation to drying in different conditions

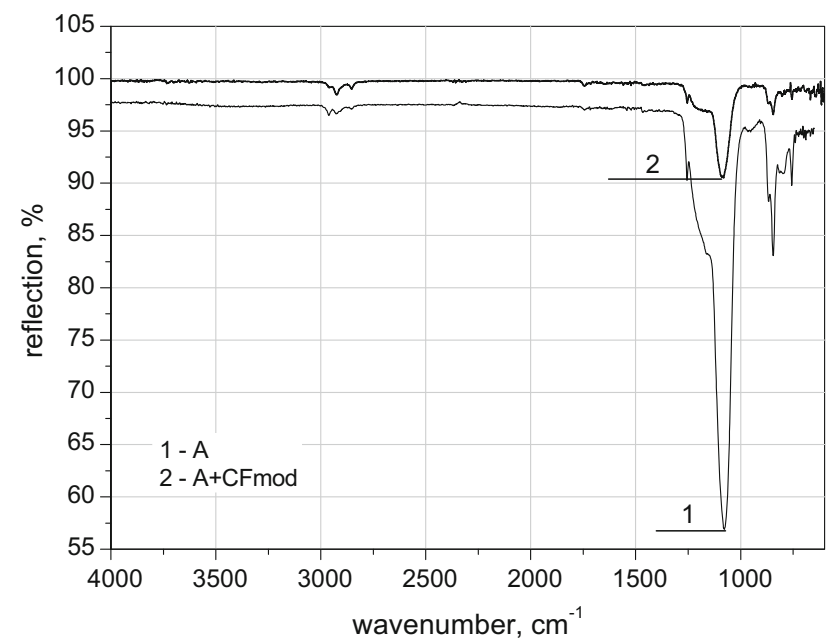

Fig. 2 FTIR analysis of pure silica aerogel and its composite with carbon fibres drying in ambient pressure

conditions, the values oscillated in the range of $0.125 \mathrm{~g} /$ $\mathrm{cm}^{3}$, whereas for the aerogels dried in atmosphere those values equalled $0.2 \mathrm{~g} / \mathrm{cm}^{3}$. Differences in density, although not so high, were also noticed for the aerogel/carbon microfiber composites, dried relatively under supercritical conditions $-0.1 \mathrm{~g} / \mathrm{cm}^{3}$ - and air-dried $-0.13 \mathrm{~g} / \mathrm{cm}^{3}$. The research also proved that the presence of carbon fibres in the aerogel structure, regardless of the way of drying, diminishes the contraction of the samples during drying resulting in three times lower contraction (in the case of drying in atmosphere) and 1.5 times lower for samples dried under supercritical conditions. Moreover, on the basis of the analysis of nitrogen adsorption isotherm with BET method, specific surface areas of the aerogels and their composites were defined, which oscillated between 775 and $863 \mathrm{~m}^{2} / \mathrm{g}$. It was noticed that the fibres influence the

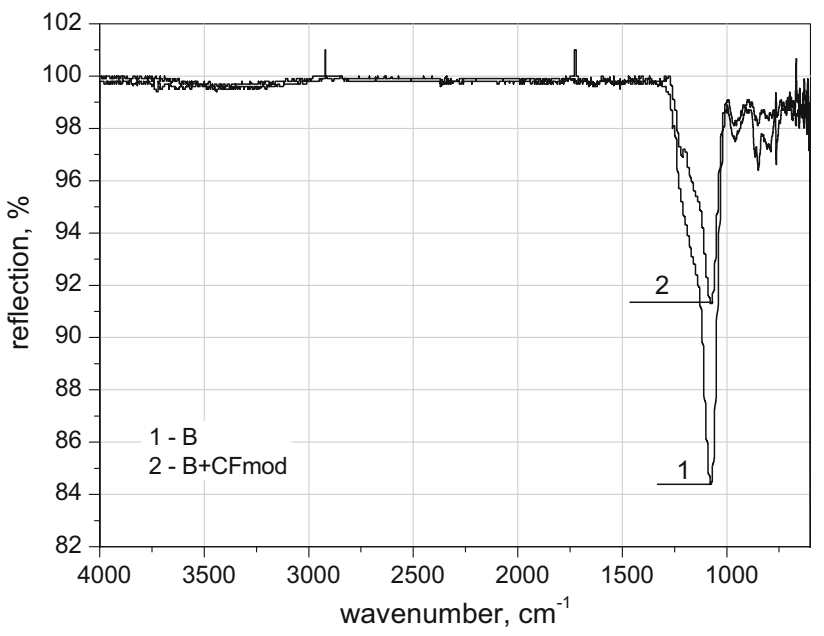

Fig. 3 FTIR analysis of pure silica aerogel and its composite with carbon fibres drying in supercritical conditions

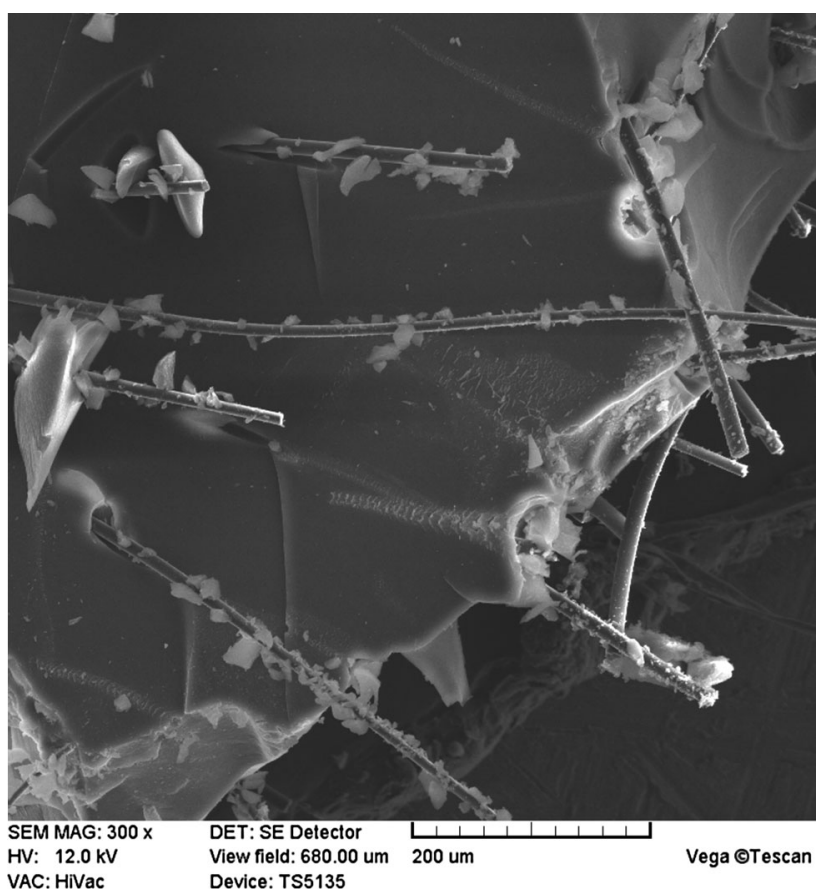

Fig. 4 SEM image of silica aerogel/carbon microfibers nanocomposites

difference in values of the specific surface area of the aerogel composites, depending on the way of drying. In the case of drying under supercritical conditions, the growth of the specific surface area was gained for the composite aerogel/carbon fibres, whereas for drying in the atmosphere, the specific surface area decreased. The way of drying had also a significant influence on the average diameter of the pores of the aerogels and their composites. Higher values of the average pore diameter $(12.2 \mathrm{~nm}-$ pure aerogel, $15.8 \mathrm{~nm}$-composite aerogel/carbon fibres) 
were gained for materials dried in atmosphere. Nevertheless, based on the IUPAC classification [7], it can be concluded that the synthesised silica aerogels and silica aerogel/carbon fibres nanocomposites maintain a porous structure and belong to mesopores materials.

Figure 1 presents the thermogravimetric curves registered for the silica aerogels and their composites with carbon microfiber dried in the atmospheric pressure and under supercritical conditions, respectively. The analysis proved that the aerogels chemically modified in the mixture of TMCS/n-hexane and air-dried had much better thermal resistance. In this case, a $2 \%$ weight loss was observed in the temperature of $200{ }^{\circ} \mathrm{C}$ and only $10 \%$ loss in $700{ }^{\circ} \mathrm{C}$. Silica aerogel/carbon fibres composites had slightly better temperature resistance in the whole research range. In the case of aerogels dried under supercritical conditions, a $2 \%$ weight loss was observed in the temperature of $100{ }^{\circ} \mathrm{C}$, whereas in higher temperatures, those materials were characterised with higher temperature stability achieving only $8 \%$ weight loss in $700{ }^{\circ} \mathrm{C}$. By contrast, the aerogel/carbon fibre composites had much lower temperature resistance in the whole research range, achieving the highest weight loss of $11 \%$ in $700{ }^{\circ} \mathrm{C}$. FTIR analysis for the aerogels and their composites, depending on the way of drying, is presented in graphs in Figs. 2 and 3. For pure aerogels, regardless of the drying conditions, by the wave number around $1100 \mathrm{~cm}^{-1}$, a high intensity peak is observed that comes from the bond $\mathrm{Si}-$ $\mathrm{O}-\mathrm{Si}[8,9]$. In the case of composites silica aerogel/carbon microfibers, in both, supercritical drying and air-drying, the intensity of this band is significantly decreased. For the materials modified in TMCS/n-hexane and air-dried, the intensity of this band is definitely the smallest. Intensity decrease in the peak coming from the $\mathrm{Si}-\mathrm{O}$ aerogel frame suggests that this bond is blocked in the structure of the silica aerogel/carbon microfibers. Oxygen functional groups present on the surface of the carbon microfibers, during the gelation of the organosilane compound, react with the hydrogen groups present on the surface of the gel, creating a fixed chemical bond [10]. This results in a significant decrease in the contraction during drying. Moreover, the research proved that the additionally conducted modification of the aerogels in the mixture of TMCS/n-hexane reinforced the structure of the pure aerogel, and especially contributes to gaining a very stable structure of the silica aerogel/carbon microfibers composite, and in this case, it is much more effective than drying under supercritical conditions.

In Fig. 4, the SEM image of silica aerogel/carbon microfiber nanocomposite was present. On the carbon fibres surface, there are visible silica aerogel particles which occurrence indicates the very good adhesion between carbon fibres and aerogel frame. Moreover, it can point out that the growth of silica gel starts on the oxidised surface of carbon microfibers.

\section{Conclusions}

As the precursor of silica aerogel in the presented work, the TEOS in the ethanol solution was applied, and as the catalyst of the gelation reaction-the hydrochloric acid. Thus, the received silica aerogels and their composites with carbon microfibers were dried under supercritical conditions and in the atmospheric pressure after the initial modification of the aerogel structure in the TMCS/nhexane mixture. The silica aerogels generated in this way were characterised by density in the range of $0.125-0.2 \mathrm{~g} /$ $\mathrm{cm}^{3}$ and specific surface areas of $801.5-863.9 \mathrm{~m}^{2} / \mathrm{g}$, relatively for those dried under supercritical conditions and those that were air-dried. In the case of pure aerogels dried under supercritical conditions, the structure presented much less contraction during drying and was more resistant to higher temperature. Moreover, the conducted research proved the advisability of the applied chemical modification of the carbon fibres. The oxygen functional groups present on the surface of the carbon microfibers, during the gelation of the organosilicon compound, reacted with the hydroxyl groups present on the gel surface, creating solid chemical bonds. This resulted in lowering the contraction during drying, both for the composites dried under supercritical conditions and in the atmospheric pressure. Moreover, the research proved that additional modification of the aerogels in the mixture of TMCS/n-hexane contributes to receiving a very stable structure of the silica aerogel/carbon microfiber composite and in this case is much more effective than drying under supercritical conditions, which was confirmed by the conducted FTIR and TG tests.

Open Access This article is distributed under the terms of the Creative Commons Attribution 4.0 International License (http://crea tivecommons.org/licenses/by/4.0/), which permits unrestricted use, distribution, and reproduction in any medium, provided you give appropriate credit to the original author(s) and the source, provide a link to the Creative Commons license, and indicate if changes were made.

\section{References}

1. Paramenter K, Milstein F (1998) J Non-Cryst Solids 223:179-189

2. Zhang Z, Shen J, Ni X, Wu G, Zhou B, Yang M, Gu X, Qian M, Wu Y (2006) J Macromol Sci Pure Appl Chem 43:1663-1670

3. Burchell TD (1999) Carbon materials for advanced technologies. Elsevier, Amsterdam 
4. Chung DDL (1994) Carbon fiber composites. ButterworthHeinemann, Newton

5. Błaszczyński T, Ślosarczyk A, Morawski M (2013) ProEng 57:200-206

6. Skowroński JM, Ślosarczyk A (2009) Przem Chem 88:823-825 (in polish)

7. de Boer JH (1958) The structure and properties of porous materials. Butterworth, London
8. Sarawade P, Kim J, Hilonga A, Quang D, Jeon S, Kim H (2011) J Non-Cryst Solids 357:2156-2162

9. Li J, Cao J, Huo L, He X (2012) Mater Lett 87:146-149

10. Venkateswara Rao A, Nielsen E, Einarsrud M-A (2001) J Non Cryst Solids 296:165-171 